\title{
Centrifugal Distortions in Molecules: An Ab Initio Approach with Application to Water
}

\author{
LAWRENCE L. LOHR \\ Department of Chemistry, University of Michigan, Ann Arbor, Michigan 48109
}

\begin{abstract}
Our procedure for employing analytical gradients of ab initio potential energy hypersurfaces in the description of centrifugally distorted molecules is applied to an asymmetric top, namely water. Both single determinantal (HF/6-31G**) and configuration-interaction (CISD/6-31G**) surfaces were utilized. Quartic centrifugal spectroscopic coefficients are obtained in both cases and are in reasonable agreement with experiment. It is shown that the calculated dependence of the energy upon the rotational angular momentum is better represented by a Padé approximant than by the conventional power series.
\end{abstract}

\section{Introduction}

In a recent publication [1] we outlined a procedure for employing analytical gradients of ab initio potential energy hypersurfaces in the description of centrifugally distorted molecules. The method is readily applicable at any computational level for which gradients are available; it does not require knowledge of second derivatives or vibrational frequencies. Stationary points are located in this structurally oriented method on the effective hypersurface defined as the sum of electronic and rotational energies. Centrifugal distortion pathways and centrifugal stabilization energies are defined; from the latter quartic centrifugal distortion spectroscopic constants were obtained in the first study [1] for $\mathrm{H}_{2}^{+}, \mathrm{NH}_{3}, \mathrm{CH}_{4}, \mathrm{BF}_{3}$, and $\mathrm{SF}_{6}$. Comparisons with experimentally determined constants were generally quite satisfactory, with calculated values being typically $10 \%$ smaller than those observed and with this error being primarily the result of the overestimation of the curvature of the electronic hypersurfaces at the computational level employed, namely $\mathrm{HF} / 6-31 \mathrm{G}^{* *}$. Particularly satisfying was the excellent description of both the scalar and tensor quartic coefficients, $D_{\mathrm{s}}$ and $D_{t}$, respectively, for the spherical tops $\mathrm{CH}_{4}$ and $\mathrm{SF}_{6}$. In a second article [2] we outlined an extension of the method to asymmetric tops and gave results for a particularly important molecule, namely ozone, for which we have also made a theoretical study of the effects of anharmonicity on its ultraviolet continuum band shape [3]. In the present article we explore two additional questions about centrifugal distortions, namely what are the effects of electron correlation on the centrifugal stabilization energies, and what is the significance of the approximately linear fall-off of effective 
quartic spectroscopic constants as observed in our earlier computations $[1,2]$ ? Both questions are approached through computational studies for another important asymmetric top, namely water, with calculations being reported at both self-consistent field (SCF) and configuration-interaction (CI) levels. The water molecule was selected because hydrides exhibit large nonrigidity effects, and because for this hydride, as well as for $\mathrm{H}_{2} \mathrm{~S}$ and $\mathrm{PH}_{3}$, it has been demonstrated [4-10] that the representation of the dependence of the rotational energy upon the angular momentum in terms of Padé approximants provides much better spectral fits than the conventional power series representation that is often divergent or poorly convergent. We noted earlier $[1,2]$ an approximately linear fall-off of our computed quartic centrifugal distortion coefficients with increasing angular momentum, this fall-off not matching the quadratic fall-off expected from the next (sextic) term in a power series representation. We demonstrate here the compatibility of an approximate linear fall-off to a Padé representation, thus providing further support for their use in spectral fittings.

In our previous studies $[1,2]$ at the $\mathrm{HF} / 6-31 \mathrm{G}^{* *}$ level we were unable to ascertain the portion of the discrepancies between calculated and observed quartic centrifugal distortion coefficients that was attributable to errors in the electronic structure calculations from the portion attributable to the limitations of our quasistatic model of centrifugal distortions. Our present example of water affords an opportunity to carry out a study utilizing analytically computed gradients at the $\mathrm{Cl}$ level, thus reducing electronic structure errors and enabling a better test of the quasistatic model. The test is severe in the case of water, for the observed [11] inertial defect $\Delta$ of $0.052 \mathrm{amu}^{2}$ (about $8.5 \%$ of the smallest moment $l_{a}$ ) indicates substantial vibrational averaging even in the ground state.

\section{Method}

We define as before $[1,2]$ an effective potential energy hypersurface $E(\mathbf{Q}, \mathbf{J})$ as

$$
E(\mathbf{Q}, \mathbf{J})=E_{\mathrm{el}}(\mathbf{Q})+E_{\mathrm{r}}(\mathbf{Q}, \mathbf{J}),
$$

where $E_{\mathrm{el}}$ denotes the electronic energy, $E_{\mathrm{r}}$ the rotational energy, $\mathbf{Q}$ the set of nuclear coordinates, and $\mathbf{J}$ the rotational angular momentum. Molecular vibration is ignored in the present form of our method, whereas molecular rotation is treated classically. Thus the method provides a description of vibrational ground states in terms of vibrationless rotating deformable bodies.

We locate stationary points on the hypersurface by the condition that $\nabla E(\mathbf{Q}, \mathbf{J})=0$. Such points are not necessarily local minima, since they may be saddle points or local maxima instead. However, for structures close to the true $(\mathbf{J}=0)$ equilibrium geometry, they have typically been found [1] to be local minima. Let $m$ be the number of internal coordinates upon which the rotational energy depends through the moment of inertia tensor. Then for a total of $n=3 N-6$ internal coordinates, where $N$ is the number of atoms in the molecule, there are $n$ equations to be solved, namely,

$$
\begin{gathered}
\partial\left(E_{\mathrm{el}}+E_{\mathrm{r}}\right) / \partial Q_{i}=0, \quad i=1, m \\
\partial E_{\mathrm{el}} / \partial Q_{j}=0, \quad j=m+1, n
\end{gathered}
$$

where $\left\{Q_{i}\right\}$ are the coordinates appearing in $E_{r}$. 
Selecting a principal axis system to describe the rotations of an asymmetric top, we write $E_{\mathrm{r}}$ simply as

$$
E_{\mathrm{r}}(\mathbf{Q}, \mathbf{J})=A(\mathbf{Q}, \mathbf{J}) J_{a}^{2}+B(\mathbf{Q}, \mathbf{J}) J_{b}^{2}+C(\mathbf{Q}, \mathbf{J}) J_{c}^{2},
$$

where $J_{a}, J_{b}$, and $J_{c}$ are the projections of the rotational angular momentum on the axes $\mathbf{a}, \mathbf{b}$, and $\mathbf{c}$. These axes are typically taken to correspond to the customary ordering of rotational constants as $A>B>C$, where these effective constants may depend on $\mathbf{J}$ as well as $\mathbf{Q}$. For water $\mathbf{a}$ is the in-plane axis perpendicular to the $C_{2}$ axis, $\mathbf{b}$ is the $C_{2}$ axis, and $\mathbf{c}$ is the axis perpendicular to the molecular plane. For asymmetric tops whose principal axis directions change with $\mathbf{J}$, it may be more convenient not to assume a principal axis representation to begin with, but for water this is not the situation. The distortions accompanying rotation about any one of the principal axes of water preserve the $C_{2 v}$ symmetry, so that there are only two structural parameters. We find it convenient to describe the molecule using the Cartesian coordinates $(x, y, z)$ for the two hydrogen atoms as $(b, \mathrm{O}, a)$ and $(b, \mathrm{O},-a)$, with the oxygen at the origin. The Cartesian axes are associated with the rotational axes according to the $I^{r}$ representation, namely $x$ with $\mathbf{b}, y$ with $\mathbf{c}$, and $z$ with $\mathbf{a}$. In terms of the distances $a$ and $b$, the bond length $R$ and bond angle $\Theta$ are given by

$$
\begin{aligned}
& R=\left(a^{2}+b^{2}\right)^{1 / 2} \\
& \Theta=2 \tan ^{-1}(a / b)
\end{aligned}
$$

The moments of inertia are

$$
\begin{aligned}
I_{a} & =2 m_{\mathrm{H}} m_{\mathrm{O}} b^{2} / M \\
I_{b} & =2 m_{\mathrm{H}} a^{2} \\
I_{c} & =I_{a}+I_{b}
\end{aligned}
$$

where $m_{\mathrm{H}}$ is the mass of an $\mathrm{H}$ atom, $m_{\mathrm{O}}$ is that of an $\mathrm{O}$ atom, and $M$ is the molecular mass.

The condition $\nabla E(\mathbf{Q}, \mathbf{J})=0$ when combined with the moments of inertia in (5) yields the following:

$\mathbf{J} \| \mathbf{a}$

$$
\begin{aligned}
\partial E_{\mathrm{el}} / \partial a & =0 \\
\partial E_{\mathrm{el}} / \partial a-J_{a}^{2} / I_{a} a & =0
\end{aligned}
$$

$\mathbf{J} \| \mathbf{b}$

$$
\begin{aligned}
\partial E_{\mathrm{cl}} / \partial a-J_{b}^{2} / I_{b} b & =0 \\
\partial E_{\mathrm{el}} / \partial b & =0
\end{aligned}
$$

$\mathbf{J} \| \mathbf{c}$

$$
\begin{aligned}
\partial E_{\mathrm{el}} / \partial a-2 m_{\mathrm{H}} a J_{c}^{2} / I_{c}^{2} & =0 \\
\partial E_{\mathrm{el}} / \partial b-2 m_{\mathrm{H}} m_{\mathrm{O}} b J_{c}^{2} / I_{c}^{2} M & =0
\end{aligned}
$$


For $\mathbf{J} \| \mathbf{a}$ we simply select $b$, obtain $a$ by criterion (6a), calculate $\partial E_{\mathrm{el}} / \partial b$ at the structure corresponding to $a$ and $b$, and then solve (6b) for $J_{a}$. The procedure for $\mathbf{J} \| \mathbf{b}$ is similar, whereas that for $J \| c$ requires a simultaneous solution of (6e) and (6f) that may be expressed by the condition that

$$
\left(\partial E_{\mathrm{el}} / \partial a\right) /\left(\partial E_{\mathrm{el}} / \partial b\right)=M a / m_{\mathrm{O}} b
$$

We define as before $[1,2]$ a centrifugal stabilization energy $\Delta E$ as the difference between the energy of a rigid molecule with $\mathbf{J}$ and that of the deformable molecule with the same $\mathbf{J}$. That is,

$$
\Delta E(\mathbf{J})=\Delta E_{\mathrm{el}}(\mathbf{J})+\Delta E_{\mathrm{r}}(\mathbf{J}),
$$

where $\Delta E_{\mathrm{el}}(\mathbf{J})$ is the negative difference between $E_{\mathrm{el}}$ at $\mathbf{Q}=\mathbf{Q}^{\circ}$, the equilibrium geometry, and at $\mathbf{Q}=\mathbf{Q}(\mathbf{J})$, the quasiequilibrium geometry for a given $\mathbf{J}$, and $\Delta E_{\mathrm{r}}$ is the positive difference between $E_{\mathrm{r}}$ at $\mathbf{Q}=\mathbf{Q}^{\circ}$ and at $\mathbf{Q}=\mathbf{Q}(\mathbf{J})$. Typically, $\Delta E_{\mathrm{r}}$ has approximately twice the magnitude of $\Delta E_{\mathrm{el}}$, so that the sum $\Delta E$ is positive with one-half the magnitude of $\Delta E_{\mathrm{r}}$.

It may appear inconsistent to assume a classical description of molecular rotation for an asymmetric top and then to consider $\mathbf{J}$ to be parallel to the intermediate principal axis b in (6c) and (6d), since such a rotation is unstable in the sense that it corresponds to a saddle point on the rotational energy surface, with intersecting contours of constant energy, corresponding to semiclassical trajectories, leading away from it. However, the Eqs. (6c) and (6d) representing the quasiequilibrium are nonetheless valid, since $\mathbf{J}$ parallel to any principal axis implies dynamical balance (unstable in some cases), with $\mathbf{J}$ parallel to the angular velocity $\boldsymbol{\omega}$. What we are so far unable to describe with our method are the dynamically unbalanced cases of $\mathbf{J}$ not parallel to a principal axis and hence not parallel to $\omega$.

The electronic structure calculations were made at two levels, both using the GAUSSIAN 82 program [12] with the split valence plus polarization basis set $6-31 \mathrm{G}^{* *}[13,14]$. The first was the single-determinental level and is designated $\mathrm{HF} /$ $6-31 \mathrm{G}^{* *}$. The second was the configuration-interaction level with all single and double excitations (including those from core levels) from a single reference configuration. This computational level is designated CISD/6-31G**. Analytical gradients were employed at each level in locating both the unconstrained $(\mathbf{J}=0)$ and constrained $(\mathbf{J} \neq 0)$ stationary points.

\section{Results and Discussion}

In Figure 1 we present in the same style used in our earlier studies $[1,2]$ the computed centrifugal stabilization energies $\Delta E$ divided by $J^{4}$ versus $J$ for the three cases $\mathbf{J}\|\mathbf{a}, \mathbf{J}\| \mathbf{b}$, and $\mathbf{J} \| \mathbf{c}$, with results being given at both the $\mathrm{HF} / 6-31 \mathrm{G}^{* *}$ and CISD/6$31 \mathrm{G}^{* *}$ levels. The equilibrium geometries differ somewhat for the two levels, with the $\mathrm{HF}$ bond angle and length $R$ being $105.9^{\circ}$ and $0.943 \AA$, whereas, the CISD values are $104.3^{\circ}$ and $0.957 \AA$; the experimental [15] equilibrium parameters are $104.5^{\circ}$ and $0.957 \AA$, respectively. From the extrapolations to $J=0$ shown by the dashed lines we obtain the quartic coefficients $D_{a}, D_{b}$, and $D_{c}$ as listed in Table I. The relationship of these parameters to the parameters $D_{J}, D_{J K}, D_{K}, \delta_{J}$, and $\delta_{K}$ is also given in Table I. 

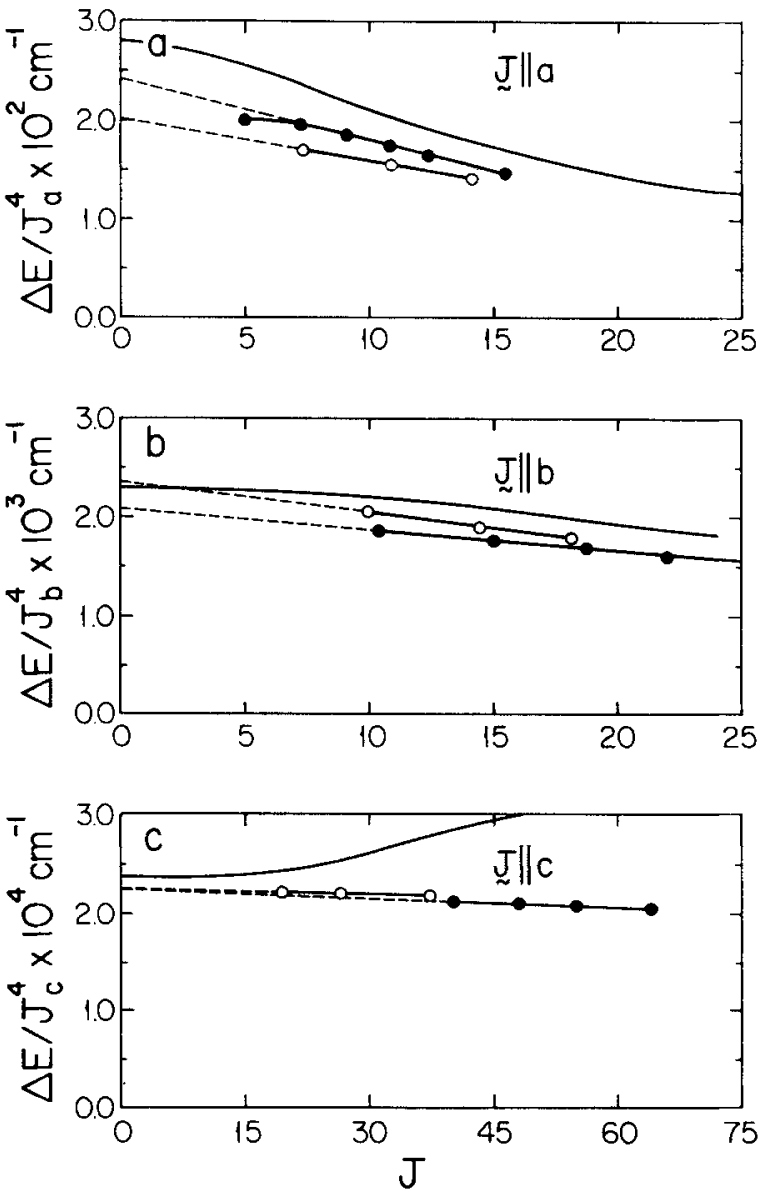

Figure 1. Centrifugal stabilization energies $\Delta E$ in centimeters ${ }^{-1}$ as defined by Eq. (8) divided by $J^{4}$ for $\mathrm{H}_{2} \mathrm{O}$ with (a) $\mathbf{J} \| \mathbf{a}$, (b) $\mathbf{J} \| \mathbf{b}$, and (c) $\mathbf{J} \| \mathbf{c}$. The open and solid circles denote values obtained at the CISD/6-31G** and $\mathrm{HF} / 6-31 \mathrm{G}^{* *}$ levels, respectively. The solid lines without points indicate values obtained using the $(1 / 2)$ Padé approximant and experimental parameters from Ref. (10).

The latter appear as coefficients in the quartic portion of the quantum mechanical rotational hamiltonian [16] (reduction $A$ ) as follows:

$$
\mathrm{H}_{4}=-D_{J} J^{4}-D_{J K} J^{2} J_{z}^{2}-D_{K} J_{z}^{4}-2 \delta_{J} J_{x y}^{2} J^{2}-\delta_{K}\left(J_{z}^{2} J_{x y}^{2}+J_{x y}^{2} J_{z}^{2}\right)
$$

where $J^{2}=\mathbf{J} \cdot \mathbf{J}, J_{x y}^{2}=J_{x}^{2}-J_{y}^{2}$, and the $x, y$, and $z$ axes correspond to $\mathbf{b}, \mathbf{c}$, and $\mathbf{a}$, respectively. The agreement with experiment [11] is generally satisfactory even at the $\mathrm{HF} / 6-31 \mathrm{G}^{* *}$ level. However, the somewhat surprising result is that the CISD/6-31G** value of $2.00 \times 10^{-2} \mathrm{~cm}^{-1}$ for $D_{a}$ (results for $\mathbf{J} \| \mathbf{a}$ extrapolated to $J=0$ ) is substantially below the observed value of $2.7952 \times 10^{-2} \mathrm{~cm}^{-1}$. This discrepancy is clearly too large to be accounted for by the error in the ab initio rotational constant $A_{\text {o }}$ (Table I), although part may arise from errors in the energies and gradients for 
TABle I. Spectroscopic constants for $\mathrm{H}_{2}^{16} \mathrm{O}$.

\begin{tabular}{lccc}
\hline Parameter $^{\mathrm{a}}$ & HF/6-31G** & CISD/6-31G** & Obs. $^{\mathrm{b}}$ \\
\hline$R$ & 0.943 & 0.957 & 0.957 \\
$\Theta$ & 105.9 & 104.3 & 104.5 \\
$A_{0}$ & 29.17 & 27.27 & 27.8806 \\
$B_{0}$ & 14.75 & 14.64 & 14.5218 \\
$C_{o}$ & 9.80 & 9.53 & 9.2777 \\
$D_{a}{ }^{c}$ & $2.40 \times 10^{-2}$ & $2.00 \times 10^{-2}$ & $2.7952 \times 10^{-2}$ \\
$D_{b}{ }^{d}$ & $2.07 \times 10^{-3}$ & $2.35 \times 10^{-3}$ & $2.2687 \times 10^{-3}$ \\
$D_{c}{ }^{\mathrm{e}}$ & $2.26 \times 10^{-4}$ & $2.30 \times 10^{-4}$ & $2.3930 \times 10^{-4}$ \\
$\nu_{1}\left(a_{1}\right)$ & 4147 & 3944 & 3657.0 \\
$\nu_{2}\left(a_{1}\right)$ & 1770 & 1710 & 1594.8 \\
$\nu_{3}\left(b_{1}\right)$ & 4264 & 4062 & 3755.8 \\
\hline \hline
\end{tabular}

${ }^{\text {a }}$ All values are given in centimeters ${ }^{1}$ except for bond length $R$ (angstroms) and bond angle $\Theta$ (degrees).

${ }^{\mathrm{b}} R$ and $\Theta$ from Ref. (15), vibrational frequencies from Ref. (18), other parameters from Ref. (11).

${ }^{\mathrm{c}} D_{a}=D_{J}+D_{J K}+D_{K}$.

${ }^{\mathrm{d}} D_{b}=D_{j}+2 \delta_{j}$.

${ }^{\mathrm{e}} D_{c}=D_{j}-2 \delta_{J}$.

nonequilibrium structures. Rather, the problem appears to lie in part in describing a molecule such as $\mathrm{H}_{2} \mathrm{O}$ with its comparatively large zero-point bending amplitude in terms of the quasistatic model. This point is discussed further below in connection with the centrifugal distortions expected for $\mathrm{D}_{2} \mathrm{O}$. We note here that in a recent comparison [17] of empirical and ab initio force fields for $\mathrm{PH}_{3}$, it was concluded that the vibrational dependence of centrifugal distortion constants may be quite large for small molecules containing light atoms.

Although vibrational frequencies are not utilized in our method for obtaining centrifugal distortion coefficients, we nonetheless computed frequencies analytically from second derivatives at the $\mathrm{HF} / 6-31 \mathrm{G}^{* *}$ level and numerically from analytic gradients at the CISD/6-31G** level (Table I). The CISD results indicate significant improvement over the HF results, although the frequencies are all still too high [18]. Although the energies and gradients we use to obtain $D$ values represent the actual HF or CI surfaces, including anharmonicities, rather than the harmonized surfaces associated with these frequencies, it is clear that there is a contribution to the errors in the $D$ values from the excessive curvature even of the cISD surface, with this error being possibly as much as $15 \%$ ( $D$ values vary inversely as the square of frequencies).

The computed centrifugal distortion pathways as bond angle changes versus bond length changes for the three cases $\mathbf{J}\|\mathbf{a}, \mathbf{J}\| \mathbf{b}$, and $\mathbf{J} \| \mathbf{c}$ may be expressed in terms of $\mathbf{J}$ for the CISD/6-31G** level as follows:
(1) $\mathbf{J} \| \mathbf{a}$
$\Delta R=8.4 \times 10^{-5} J_{a}^{2}$
$\Delta \Theta=-4.4 \times 10^{-2} J_{a}^{2}$
(2) $\mathbf{J} \| \mathbf{b}$
$\Delta R=2.9 \times 10^{-5} J_{b}^{2}$ 


$$
\begin{aligned}
& \Delta \Theta=1.5 \times 10^{-2} J_{b}^{2} \\
& \text { (3) } \mathbf{J} \| \mathrm{c} \quad \Delta R=2.2 \times 10^{-5} \mathrm{~J}_{c}^{2} \\
& \Delta \Theta \cong 0
\end{aligned}
$$

In the above $\Delta R$ is the change in bond length in $\AA, \Delta \Theta$ is the change in bond angle in degrees, and $J_{a}, J_{b}$, and $J_{c}$ are the dimensionless components of $\mathbf{J}$. The bond angle change for $\mathbf{J} \| \mathbf{c}$ is nearly zero, since the centrifugal force on each $\mathrm{H}$ atom is nearly parallel to the $\mathrm{O}-\mathrm{H}$ bond axis (exactly parallel for infinite oxygen mass). The displacements may be expressed in terms of the zero-order rotational energies using the calculated rotational constants from Table $I$ as follows:
(1) $\mathbf{J} \| \mathbf{a}$$$
\Delta R=2.9 \times 10^{-6} E_{a}^{\mathrm{o}}
$$$$
\Delta \Theta=-1.5 \times 10^{-3} E_{a}^{0}
$$$$
\text { (2) } \mathbf{J} \| \mathbf{b}
$$$$
\Delta R=2.0 \times 10^{-6} E_{b}^{\circ}
$$$$
\Delta \Theta=1.0 \times 10^{-3} E_{b}^{\mathrm{o}}
$$$$
\text { (3) } \mathbf{J} \| \mathbf{c}
$$$$
\Delta R=2.3 \times 10^{-6} E_{c}^{\circ}
$$$$
\Delta \Theta \cong 0
$$

In the above the energy components are in centimeters ${ }^{-1}$, whereas $\Delta R$ and $\Delta \Theta$ are in angstroms and degrees as before. We note, as we did for ozone [2], that the bond lengthening is roughly isotropic when expressed in terms of rotational energy rather than angular momentum.

To convert our calculated $D$ values to those appropriate for $\mathrm{D}_{2} \mathrm{O}$ is trivial for $\mathbf{J} \| \mathbf{a}$ and $\mathbf{J} \| \mathbf{b}$, as the values are simply multiplied by $\left[m_{\mathrm{H}} M\left(\mathrm{D}_{2} \mathrm{O}\right) / m_{\mathrm{D}} M\left(\mathrm{H}_{2} \mathrm{O}\right)\right]^{2} \sim 1 / 3.24$ and $\left(m_{\mathrm{H}} / m_{\mathrm{D}}\right)^{2} \sim 1 / 4$ for these two cases, respectively. However, for $\mathbf{J} \| \mathbf{c}$ the $\mathrm{D}_{2} \mathrm{O}$ distortion pathway differs from the $\mathrm{H}_{2} \mathrm{O}$ pathway; for $\mathrm{D}_{2} \mathrm{O}$, condition (7) on the electronic gradients becomes $M\left(\mathrm{D}_{2} \mathrm{O}\right) a / m_{0} b \sim 5 a / 4 b$, whereas for $\mathrm{H}_{2} \mathrm{O}$ it is $M\left(\mathrm{H}_{2} \mathrm{O}\right) a / m_{0} b \sim 9 a / 8 b$. Our cISD $/ 6-31 \mathrm{G}^{* *}$ values (Table I) for $D_{a}$ and $D_{b}$ become $6.17 \times 10^{-3}$ and $5.88 \times 10^{-4} \mathrm{~cm}^{-1}$, respectively, for $\mathrm{D}_{2} \mathrm{O}$ as compared with observed [19] values of $8.039 \times 10^{-3}$ and $5.560 \times 10^{-4} \mathrm{~cm}^{-1}$, respectively. We note that our percentage error in $D_{a}$ is less for $\mathrm{D}_{2} \mathrm{O}$ than for $\mathrm{H}_{2} \mathrm{O}$, suggesting that the smaller zero-point vibrational amplitudes in $\mathrm{D}_{2} \mathrm{O}$ make our quasistatic model more accurate for it than for $\mathrm{H}_{2} \mathrm{O}$. The centrifugal displacements for a given $\mathbf{J}$ are also smaller for $\mathrm{D}_{2} \mathrm{O}$ than for $\mathrm{H}_{2} \mathrm{O}$; for $\mathbf{J} \| \mathbf{a}$ and $\mathbf{J} \| \mathbf{b}$ they are simply the $\mathrm{H}_{2} \mathrm{O}$ values multiplied by $\left[m_{\mathrm{H}} M\left(\mathrm{D}_{2} \mathrm{O}\right) / m_{\mathrm{D}} M\left(\mathrm{H}_{2} \mathrm{O}\right)\right]^{1 / 2} \sim 1.8^{-1 / 2}$ and $\left(m_{\mathrm{H}} / m_{\mathrm{D}}\right)^{1 / 2} \sim 2^{-1 / 2}$, respectively.

The nearly linear fall-off with increasing $J$ of the $\Delta E / J^{4}$ values in Figure 1 closely resembles the behavior we previously found $[1,2]$ for other molecules. If sextic and no higher terms in the dependence of the energy on the angular momentum were important, a parabolic fall-off would be expected. The sextic spectroscopic coefficient could then be obtained from the slope of $\Delta E / J^{4}$ versus $J^{2}$ rather than $J$. As noted above, it has been demonstrated [4-10] for $\mathrm{H}_{2} \mathrm{O}$ and other hydrides $\left(\mathrm{H}_{2} \mathrm{~S}\right.$ and $\left.\mathrm{PH}_{3}\right)$ that the use of Padé approximants to represent the dependence of the energy upon angular momentum is greatly superior to the use of conventional power series. Various 
forms of approximants have been used [4-10, 20], with a particularly simple form being that recently employed in describing $\mathrm{H}_{2} \mathrm{D}^{+}, \mathrm{HD}_{2}^{+}$, and $\mathrm{D}_{3}^{+}$, namely

$$
H_{6}^{p}=\left(H_{4}\right)^{2} /\left(H_{4}-H_{6}\right)
$$

where $H_{4}$ is the quartic Hamiltonian, such as that from (9), and $H_{6}$ is the analogous [16] sextic Hamiltonian. Corresponding to (12) there is an effective $\Delta E / J^{4}$ that varies as $J^{-2}$ for sufficiently large $J$ and thus approaches zero as $J$ approaches infinity. However Padé approximants with polynomials of different degrees in the numerator and/or denominator will in general have different limiting behavior; if the numerator is of a higher degree than the denominator by $J^{4}$, then $\Delta E / J^{4}$ approaches a constant different from $D$ as $J$ approaches infinity. A particularly effective approximant is the $(1 / 2)$ form used by Polyanski [10] to fit the rotational levels of $\mathrm{H}_{2} \mathrm{O}$; constructed from quartic, sextic, and octic terms of a power series it, like (12), gives $\Delta E / J^{4}$ varying as $J^{-2}$ for large $J$. In Figure 1 the solid lines represent $\Delta E / J^{4}$ values using this form together with Polyanski's parameters that differ only slightly from the values of DeLucia et al. [11]. Although no rigorous comparison is made, the quasilinear behavior of $\Delta E / J^{4}$ as obtained from the Padé representation is apparent. [For $\mathbf{J} \| \mathbf{c}$, this particular Padé representation becomes inadequate at the high $J$ values $(>20)$ considered here.]

\section{Summary}

Centrifugal distortion coefficients have been obtained for $\mathrm{H}_{2} \mathrm{O}$ using our quasistatic model $[1,2]$ at the $\mathrm{HF} / 6-31 \mathrm{G}^{* *}$ and $\mathrm{CISD} / 6-31 \mathrm{G}^{* *}$ levels. The CISD values are closer to the observed than the HF values for $\mathbf{J} \| \mathbf{b}$ and $\mathbf{J} \| \mathbf{c}$. However, for $\mathbf{J} \| \mathbf{a}$, where $\mathbf{a}$ is the in-plane axis perpendicular to the $C_{2}$ axis, the CISD value is approximately $28 \%$ too small. The centrifugal distortion involves more of a change in bond angle for this direction of $\mathbf{J}$ than for any other principal direction. Thus, the result is especially sensitive not only to error in the bending frequency (implicitly incorporated in our method via gradients) but also to vibrational averaging effects expected to be significant for hydrides.

\section{Acknowledgments}

The author wishes to thank Mr. A. Taleb-Bendiab for assistance with the calculations and the University of Michigan Computing Center for the use of its facilities.

\section{Bibliography}

[1] L. L. Lohr and J.-M. J. Popa, J. Chem. Phys. 84, 4196 (1986).

[2] L. L. Lohr and A. J. Helman, J. Comp. Chem. 8, 307 (1987).

[3] L. L. Loht and A. J. Helman, J. Chem. Phys. 86, 5329 (1987).

[4] S. P. Belov, A. V. Burenin, O. L. Polyansky, and S. M. Shapin, J. Mol. Spectrosc. 90, 579 (1981).

[5] A. V. Burenin, O.L. Polyanskii, and S. M. Shchapin, Opt. Spectrosc. (Engl. Transl.) 53, 395 (1982).

[6] A. V. Burenin, T. M. Fevral'skikh, E. N. Karyakin, O. L. Polyansky, and S. M. Shapin, J. Mol. Spectrosc. 100, $182(1983)$ 
[7] A. V. Burenin, O. L. Polyanskii, and S. M. Shchapin, Opt. Spectrosc. (Engl. Transl.) 54, 256 (1983).

[8] A. V. Burenin and V1. G. Tyuterev, J. Mol. Spectrosc. 108, 153 (1984).

[9] A. V. Burenin, T. M. Fevral'skikh, A. A. Mel'nikov, and S. M. Shapin, J. Mol. Spectrosc. 109, 1 (1985).

[10] O.L. Polyansky, J. Mol. Spectrosc. 112, 79 (1985).

[11] F. C. DeLucia, P. Helminger, and W. H. Kirchhoff, J. Phys. Chem. Ref. Data 3, 1 (1974).

[12] J. S. Binkley, M. J. DeFrees, R. Krishnan, R. A. Whiteside, B. S. Schlegel, E. M. Fluder, and J. Pople, GAUSSIAN 82 (Carnegie-Mellon University, Pittsburgh, Pa., 1983).

[13] W. J. Hehre, R. Ditchfield, and J. A. Pople, J. Chem. Phys. 56, 2257 (1972).

[14] R. C. Hariharan and J. A. Pople, Theor. Chim. Acta 28, 213 (1973).

[15] W. S. Benedict, N. Gailar, and E. K. Plyler, J. Chem. Phys. 24, 1139 (1956).

[16] J. K. G. Watson, Vibrational Spectra and Structure, J. R. Durig, Ed. (Elsevier, Amsterdam, 1977), Vol. 6, pp. 2-89.

[17] J. L. Duncan and D. C. McKean, J. Mol. Spectrosc. 107, 301 (1984).

[18] G. Herzberg, Molecular Spectra and Molecular Structure, III. Electronic Spectra and Electronic Structure of Polyatomic Molecules (Van Nostrand, Princeton, NJ, 1966), p. 585.

[19] G. Steenbeckeliers and J. Bellet, J. Mol. Spectros. 45, 10 (1973); also, see W. S. Benedict, S. A. Clough, L. Frenkel, and T. E. Sullivan, J. Chem. Phys. 53, 2565 (1970).

[20] A. V. Burenin, J. Mol. Spectrosc. 115, 163 (1986).

[21] S. C. Foster, A. R. W. McKellar, I. R. Peterkin, J. K. G. Watson, F. S. Pan, M. W. Crofton, R. S. Altman, and T. Oka, J. Chem. Phys. 84, 91 (1986).

[22] S. C. Foster, A. R. W. McKellar, and J. K. G. Watson, J. Chem. Phys. 85, 664 (1986).

[23] J. K. G. Watson, S. C. Foster, and A. R. W. McKellar, Can. J. Phys. 65, 38 (1987).

Received March 16, 1987. 